\title{
Erratum to: Detecting small lung tumors in mouse models by refractive-index microradiology
}

\author{
Chia-Chi Chien • Guilin Zhang • Yeukuang Hwu • Ping Liu • Weisheng Yue • \\ Jianqi Sun • Yan Li • Hongjie Xu • Lisa X. Xu • Chang Hai Wang • Nanyow Chen • \\ Chien Hung Lu • Ting-Kuo Lee $\cdot$ Yuh-Cheng Yang • Yen-Ta Lu • Yu-Tai Ching • \\ T. F. Shih • P. C. Yang • J. H. Je • Giorgio Margaritondo
}

Published online: 13 July 2011

(C) Springer-Verlag 2011

\section{Erratum to: Anal Bioanal Chem \\ DOI 10.1007/s00216-011-5117-x}

We should like to call your attention to the fact that Hongjie $\mathrm{Xu}$ 's name was misspelled in the original publication.

The correct list of authors for this contribution is: Chia-Chi Chien $^{1,3}$, Guilin Zhang ${ }^{2}{ }^{凶}$, Y. Hwu ${ }^{1,3}$, Ping Liu ${ }^{4}$, Weisheng $\mathrm{Yue}^{2}$, Jianqi $\mathrm{Sun}^{4}$, Yan $\mathrm{Li}^{2}$, Hongjie $\mathrm{Xu}^{2}$, Lisa $\mathrm{X} . \mathrm{Xu}^{4}$, Chang Hai Wang ${ }^{1}$, Nanyow Chen ${ }^{1}$, Chien Hung $\mathrm{Lu}^{1}$, TingKuo Lee ${ }^{1}$, Yuh-Cheng Yang ${ }^{5}$, Yen-Ta Lu ${ }^{5}$, Yu-Tai Ching ${ }^{6}$, T. F. Shih ${ }^{7}$, P. C. Yang ${ }^{7}$, J. H. Je ${ }^{8}$ and G. Margaritondo ${ }^{9}$

The online version of the original article can be found at http://dx.doi. org/10.1007/s00216-011-5117-x.

C.-C. Chien $\cdot$ Y. Hwu $\cdot$ C. H. Wang $\cdot$ N. Chen $\cdot$ C. H. Lu $\cdot$

T.-K. Lee

Institute of Physics, Academia Sinica,

Nankang,

Taipei 11529, Taiwan

G. Zhang $(\bowtie) \cdot$ W. Yue $\cdot$ Y. Li $\cdot$ H. Xu

Shanghai Institute of Applied Physics,

Chinese Academy of Sciences,

Shanghai 201800, China

e-mail: zhangguilin@sinap.ac.cn

\section{C.-C. Chien • Y. Hwu}

Department of Engineering and System Science,

National Tsing Hua University,

Hsinchu 30013, Taiwan

P. Liu $\cdot$ J. Sun $\cdot$ L. X. Xu

Shanghai Jiao Tong University,

Shanghai 200030, China
Y.-C. Yang • Y.-T. Lu

Mackay Memorial Hospital,

Taipei City 104, Taiwan

Y.-T. Ching

Department of Computer Science,

National Chiao Tung University,

Hsinchu 300, Taiwan

T. F. Shih $\cdot$ P. C. Yang

College of Medicine, National Taiwan University,

Taipei 10617, Taiwan

J. H. Je

X-ray Imaging Center,

Pohang University of Science and Technology Pohang,

Pohang CT,

Kyungbuk 790-784, South Korea

G. Margaritondo

Ecole Polytechnique Fédérale de Lausanne (EPFL),

1015 Lausanne, Switzerland 